psychopraxis.neuropraxis $2017 \cdot 20: 203-207$ https://doi.org/10.1007/s00739-017-0420-8 Online publiziert: 5. September 2017 @ ( Der/die Autor(en) 2017. Dieser Artikel ist eine Open-Access-Publikation.

CrossMark

\author{
Annemarie Unger ${ }^{1}$ Thomas Foki ${ }^{2}$ Gabriele Sachs ${ }^{1}$ \\ 'Universitätsklinik für Psychiatrie und Psychotherapie, Klinische Abteilung für Sozialpsychiatrie, \\ Medizinische Universität Wien, Wien, Österreich \\ ${ }^{2}$ Universitätsklinik für Neurologie, Medizinische Universität Wien, Wien, Österreich
}

\title{
Einsatz von Antipsychotika bei Parkinson-Psychose und Multimorbidität
}

\section{Überblick und Kasuistik}

\section{Einleitung}

Die Angaben zur Prävalenz von psychotischer Symptomatik (in der herkömmlichen Definition Halluzinationen und/oder Wahnvorstellungen) beim primären Parkinsonsyndrom variieren stark (zwischen 30 und $60 \%$ ), und ihre Häufigkeit hat mit der Entwicklung der dopaminergen Antiparkinsonika erheblich zugenommen [1-3]. Psychotische Symptome können als einfache optische Halluzinationen bei voller Einsicht bis hin $\mathrm{zu}$ schwerer paranoider Wahnsymptomatik auftreten und stellen die Angehörigen vor große Herausforderungen [4]. Das Auftreten von psychotischen Symptomen ist einerseits bedingt durch intrinsische Faktoren, andererseits werden sie auch durch äußere Faktoren wie z. B. durch die Behandlung mit dopaminergen Substanzen begünstigt, hier mehr von Dopaminagonisten als durch Monotherapie mit Levodopa [5]. Ihr Auftreten führt zwangsläufig $\mathrm{zu}$ einer Einschränkung der Anti-Parkinson-Therapie mit Verschlechterung der motorischen Situation, aber auch vermehrt zu einer Verschlechterung des Krankheitsverlaufs. Risikofaktoren für das Auftreten von psychotischen Symptomen sind kognitive Einschränkungen bzw. Demenz (Lewy-Körperchen-Pathologie), psychiatrische Komorbidität (wie z. B. Alkoholabhängigkeit, Depression), Patientenalter, Dauer und Schwere der Parkinson-Erkrankung, Sehstörun- gen, eine ungewohnte Umgebung und Schlafstörungen (• Tab. 1 und 2).

\section{\) Parkinson-Patienten sind häufig multimorbid und entwickeln oft psychotische Symptome}

Eine hohe anticholinerge Last durch Medikation wirkt sich im Zusammenspiel mit krankheitsbedingten Unterbrechungen von cholinerger Konnektivität ungünstig aus [6]. Levodopa, Pramipexol, Selegilin, Entacapon und vor allem Amantadin haben leichte anticholinerge Effekte, sind jedoch mehrheitlich in der Behandlung des Morbus Parkinson unabkömmlich.

\section{Therapie der Parkinson- Psychose}

Der Einsatz von Antipsychotika bei M. Parkinson sollte generell sehr zurückhaltend erfolgen. Nicht nur sind Parkinson-Patienten bezüglich des Auftretens von extrapyramidaler Symptomatik viel vulnerabler als Schizophreniepatienten, der Einsatz von Antipsychotika bei M. Parkinson ist generell mit einer erhöhten Mortalität vergesellschaftet [7].

\section{Clozapin}

Zur klinischen Wirksamkeit bei Parkinson-Psychose gibt es für Clozapin
(6,25-50 mg TD) vs. Plazebo die beste Evidenzlage mit mehreren randomisiert kontrollierten Studien [5, 8, 9], jedoch ist Clozapin mit dem gravierenden Nachteil eines Agranulozytoserisikos von 1-2\%behaftet (wöchentliche Blutbildkontrollen für die ersten 18 Wochen erforderlich, danach monatlich) und hat ein anticholinerges Potenzial, welches den Einsatz aufgrund der möglichen Induktion/Aufrechterhaltung von Delirien einschränkt.

\section{Quetiapin}

In seinen Eigenschaften dem Clozapin ähnlich ist Quetiapin, welches aktuell am häufigsten zum Einsatz kommt (12,5 mg bis max. $150 \mathrm{mg}$ ). Quetiapin ist im allgemeinen gut verträglich, sollte wegen seiner sedierenden Eigenschaften als Einmalgabe abends verordnet werden, be-

\section{Tab. 1 Risikofaktoren für Delir}

- Kognitive Beeinträchtigung oder Demenz

- Alter >65 Jahre

- Multimorbidität

- In der Anamnese Delir, Insult, neurologische Erkrankung, Stürze oder Bewegungsstörung

- Verwendung von Psychopharmaka

- Polypharmazie (>4 Medikamente)

- Schlafstörungen

- Spitalaufenthalt/ Intensivstationsaufenthalt/ungewohnte Umgebung, Operation

- Fehlendes Hörgerät/fehlende Brille 
ginnend mit $12,5 \mathrm{mg}$ und nur langsam (wöchentlich) in der Dosis gesteigert werden, je nach klinischem Bild. Es hat ebenfalls ein Agranulozytoserisiko, jedoch ein geringeres, und ist ebenfalls anticholinerg wirksam (durch seine Metaboliten). Für Quetiapin gibt es bis dato acht offene Studien mit insgesamt 191 Patienten, wobei bei 152 (80\%) eine Besserung durch die Behandlung beobachtet werden konnte [2]. Des Weiteren gibt es vier Vergleichsstudien Quetiapin vs. Plazebo, wobei jedoch bei dreien [10-12] keine signifikanten Unterschiede in Hinblick auf psychotische Symptome gefunden wurden. In einer der Studien [13] wurde zwar eine Besserung der psychotischen Symptomatik und des CGI (clinical global impression) gefunden, jedoch bei eingeschränkter Aussagekraft bedingt durch eine sehr kleine Samplegröße von $n=11$ [3].

\section{Clozapin vs. Quetiapin}

Zusätzlich gab es zwei einfach verblindete randomisiert kontrollierte Vergleichsstudien Clozapin vs. Quetiapin [14, 15]. Diese Studien zeigten keine signifikanten Unterschiede hinsichtlich der motorischen Situation, wobei bei der Studie von Morgante et al. die Anti-ParkinsonTherapie unverändert weitergeführt wurde, während bei der Studie von Merims et al. für mindestens 2 Wochen im Vorfeld Selegilin, Amantadin und anticholinerge Substanzen pausiert wurden.

\section{Olanzapin}

Für Olanzapin vs. Plazebo gibt es ebenfalls drei Studien [16-18], welche jedoch sämtlich keine signifikanten Ergebnisse in Hinblick auf psychotische Parameter erbrachten, sodass Olanzapin nicht empfohlen werden kann [3].

\section{Pimavanserin}

Eine bislang in Österreich noch nicht erhältliche neue Option in der Behandlung von psychotischer Symptomatik bei Parkinson stellt Pimavanserin dar, ein nondopaminerges atypisches Antipsychotikum, welches erstmalig 2016 von der FDA in den Vereinigten Staaten für die- se Indikation zugelassen wurde. Es hat einen innovativen Wirkmechanismus, da es als selektiver inverser Agonist am Serotonin-5HT-2A-Rezeptor wirkt. Bis dato gibt es zwei Studien, welche die Effektivität von Pimavanserin vs. Plazebo belegen $[19,20]$. In Anbetracht der Datenlage lässt sich feststellen, dass Pimavanserin künftig eine First-line-Therapie für Parkinson-Psychose sein könnte.

Der Einsatz von Antipsychotika ist in mehrfacher Hinsicht problematisch nicht nur aufgrund von extrapyramidaler Symptomatik, sondern auch bei Polypharmazie und internistischer Multimorbidität aufgrund der kardialen Problematik mit Verlängerung der QTc-Zeit [21]. Das Auftreten einer QTc-Zeit-Verlängerung hängt unter anderem von der Nierenfunktion, dem Geschlecht und der basalen QTc-Zeit sowie der Komedikation ab [22]. Haloperidol kann die QTcZeit verlängern und schwere Herzrhythmusstörungen auslösen, dieses gilt vor allem für höhere Dosierung sowie bei intravenöser Injektion. In Österreich besteht keine Zulassung für die i. v. Applikation von Haloperidol [23]. Das relative Risiko einer QTc-Zeit-Verlängerung bei atypischen Antipsychotika ist am höchsten für Ziprasidon $>$ Quetiapin $=$ Risperidon $=$ Olanzapin. Aripiprazol ist ebenfalls mit einem geringeren Risiko vergesellschaftet. Clozapin kann dosisabhängig zu einer Verlängerung des QTc-Intervalls führen, ähnlich wie Olanzapin und Haloperidol.

Auftreten von extrapyramidaler Symptomatik ist am wahrscheinlichsten beim Einsatz von hochpotenten Neuroleptika (NL) der ersten Generation z. B. Haloperidol > mittelpotente ErstgenerationNeuroleptika $=$ Risperidon $>$ niederpotentes NL erste Generation > Olanzapin $=$ Ziprasidon $>$ Quetiapin $>$ Clozapin [24]. Bei jenen Patienten, welche zusätzlich eine Demenz aufweisen, sollten Acetylcholinesterasehemmer (z. B. Rivastigmin) zum Einsatz kommen, da diese auch zu einer Besserung der psychotischen Symptomatik beitragen können [25]. psychopraxis.neuropraxis $2017 \cdot 20: 203-207$ https://doi.org/10.1007/s00739-017-0420-8 @ ( Der/die Autor(en) 2017. Dieser Artikel ist eine Open-Access-Publikation.

\section{A. Unger $\cdot$ T. Foki $\cdot$ G. Sachs}

Einsatz von Antipsychotika bei Parkinson-Psychose und Multimorbidität. Überblick und Kasuistik

\section{Zusammenfassung}

Parkinson-Patienten sind häufig multimorbid und haben ein hohes Risiko, psychotische Symptome oder ein Delir zu entwickeln. Die Therapie der psychotischen Symptome mit Antipsychotika ist mit Einschränkungen behaftet. Wir berichten über einen 62-jährigen Patienten, welcher im Zuge des Konsiliardienstes während zwei Aufenthalten an der Unfallchirurgie mehrfach begutachtet wurde.

Schlüsselwörter

Psychose - Morbus Parkinson - Antipsychoti$\mathrm{ka} \cdot$ Anticholinergika $\cdot$ Multimorbidity

\section{Use of antipsychotics in the therapy of Parkinson's psychosis and multimorbidity. Overview and case report}

\section{Abstract}

Parkinson's disease patients frequently suffer from numerous comorbid conditions and have a high risk of developing psychotic symptoms or delirium. Treatment of psychotic symptoms in Parkinson's patients is restricted by side effects. We present a 62 year-old patient who was seen several times by psychiatrists and neurologists at the department for trauma surgery.

Keywords

Psychosis - Parkinson's disease . Antipsychotics · Anticholinergic drugs . Multimorbidity

\section{Kasuistik \\ Erster stationärer Aufenthalt}

Wir berichten über einen 62-jährigen Patienten, der im Rahmen des psychiatrischen Konsiliardienstes auf der Unfallchirurgie mehrfach begutachtet wurde. Bekannt waren ein linksbetontes primäres Parkinson-Syndrom (Amantadin- 
sulfat 100 mg 1-0-0-0), Osteoporose, paroxysmales Vorhofflimmern, Nikotinabhängigkeit (60/die) sowie eine bipolar affektive Störung (Lamotrigin TD $200 \mathrm{mg}$ ). Anamnestisch bestand eine Alkoholabhängigkeit, jedoch war der Patient zum Aufnahmezeitpunkt schon seit mehreren Jahren nachweislich abstinent. Im April 2016 stürzte er aufgrund von Parkinson-bedingten Gleichgewichtsstörungen zu Hause und zog sich Wirbelkompressionsfrakturen im Bereich Th12 und L4 zu. Klinisch zeigten sich keine neurologischen Ausfälle im Zusammenhang mit dem Trauma. Nach bildgebenden Abklärungen (Röntgen-, CT- und MRT-Aufnahmen) wurde eine operative Versorgung beschlossen. Zum Aufnahmezeitpunkt nahm der Patient kein Antipsychotikum, keine Schlafmedikation und lehnte die Applikation eines Nikotinpflasters ab. Von organischer Seite fanden sich im CCT zum Aufnahmezeitpunkt keine Auffälligkeit und keine Befunddynamik im Vergleich zu einem Vor-CCT, welches leider für diesen Fallbericht nicht zugänglich war.

Im Aufnahmelabor fanden sich eine leichte CRP-Erhöhung und eine Leukozytose. Nachträglich lässt sich spekulieren, dass die Entwicklung des Delirs ursächlich mit dem inzipienten Infekt, dem Nikotinentzug sowie dem Ortswechsel (Spitalsaufenthalt) in Zusammenhang stehen könnte. Der Patient wurde vor der geplanten OP zunehmend desorientiert, logorrhoisch, aggressiv und optisch halluzinierend (Gestalten vor dem Fenster) bei schwankender Vigilanz und Aufmerksamkeit. In der Nacht war er stationsflüchtig, delirant, in der Früh zeigte sich nach Monitoring ein paroxysmales tachykardes Vorhofflimmern ohne kardiorespiratorische Symptomatik. Mit der Gabe von $300 \mathrm{mg}$ Amiodaronhydrochlorid i.v. konnte eine prompte Kardioversion in einen normofrequenten Sinusrhythmus erzielt werden. Der Patient berichtete, in der Nacht seine Schwester gesehen zu haben mit welcher er geredet habe, fragte nach bezüglich eines Skigebietes, dieses könne er von seinem Bett aus sehen. Des Weiteren bestand eine paranoide Reaktionsbereitschaft, Pat. hatte Sorge, an der Station eingesperrt zu werden oder von einer Kir- che angeworben zu werden. Pat. erhielt Quetiapin 25 mg 0-0-0-1. Aufgrund der erhöhten Entzündungsparameter wurde eine Infektabklärung durchgeführt.

Vom Konsiliararzt der Neurologie wurde das Einsetzen einer Levodopaund Benserazid-Therapie in Kombination mit Amantadinsulfat in halbierter Dosis empfohlen. Mit Flüssigkeitsgabe, Kaliumsubstitution, Antibiose sowie Neueinstellung der Antiparkinsontherapie konnte eine deutliche Besserung des Allgemeinzustands und kognitiven Status erreicht werden. Nach Rücksprache mit dem Internisten der Klinik wurde eine Dauertherapie mit einem Betablocker in niedriger Dosis begonnen, das Präparat wegen Entwicklung einer asymptomatischen Bradykardie später langsam abgesetzt; bei einem Chad-Vasc-Score von 0 wurde von einer Antikoagulation Abstand genommen. Der Allgemeinzustand des Patienten besserte sich so weit, dass er operativ versorgt werden konnte: Kyphoplastie, dorsale Stabilisierung mit Horizon-
Longitude; die Operation verlief komplikationslos. Postoperativ konnte der Patient relativ schnell mobilisiert werden, zum Zeitpunkt der Entlassung war er selbstständig mit zwei Krücken und gelegentlich mit Begleitperson mobil. Ein pseudoradikulärer Schmerz, am ehesten Clunealnerven entsprechend, wurde vom Konsiliarneurologen mit Neurontin in steigender Dosis behandelt. Die Operationswunde war vollkommen bland, die Nähte bereits entfernt, die Röntgenkontrollen postoperativ und nach Mobilisierung zeigten einen regelrechten Sitz der osteosynthetischen Implantate. Die Antibiose konnte nach 11 Tagen Gabe beendet werden, als analgetische Therapie erhielt der Patient zuletzt Diclobene in Kombination mit Metamizol. Der Patient konnte aus der stationären Behandlung entlassen werden.

\section{Zweiter stationärer Aufenthalt}

Ein halbes Jahr später stürzte der Patient erneut und zog sich eine geschlossene
Hier steht eine Anzeige.

\section{Springer}




Tab. 2 Medikamente welche potenziell
delirogen wirken können (Auszug)
- Alle anticholinerg wirksamen Medika-
mente (Atropin, Biperiden, Oxybutinin)
- Analgetika, auch NSAR, Opiate
- Antibiotika (Chinolone, Gentamycin,
Isoniazid)
- Antidepressiva
- Antihistaminika
- Antihypertensiva (Clonidin, Hydralazin,
Propranolol, Reserpin)
- Anti-Parkinson-Medikamente
- Benzodiazepine
- Diuretika
- Digitalis
- Glukokortikoide: Cortison u. Ä.
- Insulin, Sulfonylharnstoffe
- Lithium
- Neuroleptika (Clozapin, Olanzapin Levo-
mepromazin, Promethazin, Perphenazin)
- Urologika: Inkontinenzmedikamente

Fraktur des Os pubis ramus sup. et inf. zu. Zur Schmerztherapie und Mobilisierung wurde er erneut stationär aufgenommen. Aufgrund der Schmerzen erhielt der Patient Fentanyl-Depotpflaster $75 \mu \mathrm{g} / 72 \mathrm{~h}$ und konservative Physiotherapie zwecks Mobilisierung. Aufgrund des Umspringens von paroxysmalem Vorhofflimmern (VHF) in ein dauerhaftes VHF wurde Pat. vollheparinisiert mit $2 \times 80 \mathrm{mg}$ Enoxaparin-Natrium. Im Zuge des Aufenthalts kam es - möglicherweise durch Fentanyl - zu einer zunehmenden Verschlechterung des Allgemeinzustands mit Delir, optischen Halluzinationen, Unruhe und Nesteln. Von einem Antipsychotikum wurde aufgrund der kardialen $\mathrm{Si}$ tuation vorübergehend Abstand genommen, der Patient erhielt zwecks Sedierung Lorazepam bis zu $4 \mathrm{mg}$ TD.

\section{》) Auslöser eines Delirs kann jede Anti-Parkinson-Therapie sein}

Des Weiteren erfolgte eine Umstellung des Schmerzpflasters von Fentanyl auf Buprenorphin. Der Patient entwickelte eine Akinesie, die orale Medikation mit Amantadinsulfat $200 \mathrm{mg}$ TD wurde auf intravenös umgestellt und die Do- sis trotz Bradykardiegefahr auf bis zu $400 \mathrm{mg}$ TD gesteigert. Im Neurostatus fand sich ein links betontes Parkinson-Syndrom mit Hypodiadochokinese, Feinmotilitätsstörung, Rigor und $\mathrm{Ru}$ he- sowie Intentionstremor. Es kam $\mathrm{zu}$ einer kontinuierlichen Verschlechterung des Allgemeinzustands mit respiratorischer Insuffizienz, sodass eine Transferierung von der Normalstation auf die Intensivstation erfolgte, wo der Patient tracheotomiert und beatmet wurde. Eine Antibiose wurde begonnen, nachdem in der Blutkultur Streptococcus pneumoniae nachgewiesen wurde. Der Patient hatte nachher kurzfristig eine Clostridium-difficile-positive Diarrhoe. Amantadinsulfat wurde pausiert, von neurologischer Seite wurde (da weniger Psychose-auslösendes Potenzial) Levodopa/Benserazid zunächst $3 \times$ tgl. etabliert. Zusätzlich wurde mit Rotigotin-Transdermalpflaster $8 \mathrm{mg} / 24 \mathrm{~h}$ begonnen. Aufgrund der weiterhin bestehenden psychomotorischen Agitation mit Halluzinationen wurde von psychiatrischer Seite Seroquel $50 \mathrm{mg}$ 0-0-0-1 etabliert. Nach Beherrschung der septischen Situation, der respiratorischen Probleme sowie neuropsychiatrischer Besserung konnte der Patient dekanüliert werden. Ab diesem Zeitpunkt konnte eine progressive Besserung dokumentiert werden. Es erfolgte ein Rücktransfer an die Normalstation, und der Pat. konnte schrittweise mobilisiert werden. Letztlich wurde die Anti-Parkinson-Therapie auf Wunsch des Patienten nochmal optimiert. Somit ergab sich folgende Entlassungsmedikation: Levodopa/Carbidopa 200/50 mg 0-0-0-1, Rotigotin transdermales Pflaster $8 \mathrm{mg} / 24 \mathrm{~h} 1 \times$ tgl., Levodopa/Benserazid 100/25 mg $8 \mathrm{~h}, 12 \mathrm{~h}$, $16 \mathrm{~h}, 20 \mathrm{~h}$, Amiodaron $200 \mathrm{mg}$ 1-0-10 , Lamotrigin $100 \mathrm{mg}$ 1-0-1-0, Quetiapin $50 \mathrm{mg}$ 0-0-0-1, Tri-Kalium-citrat/ Kaliumhydrogencarbonat Btl. 1-0-10 , Magnesiumcarbonat/Magnesiumoxid Btl. 1-0-1-0, Metamizol 20 gtt 3-4 tgl. bei Schmerzen, Fenoterolhydrobromid/ Ipratropiumbromid-Hübe bei Bedarf. Im neurologischen Konsil ergab sich aufgrund der reduzierten Auffassungsleistung und der mnestischen Defizite der Verdacht auf ein beginnendes demenzielles Syndrom (womöglich auch Lewy-
Körper-Demenz?), wobei aufgrund des im Vordergrund stehenden schlechten Allgemeinzustands während des Aufenthalts eine weitere Abklärung zum Entlassungszeitpunkt noch ausständig war. Eine neuropsychologische Testung wurde empfohlen, eventuell in weiterer Folge je nach Befund Etablierung eines Acetylcholinesterasehemmers.

\section{Fazit für die Praxis}

Vorgehen bei Auftreten eines Parkinson-Delirs nach J. Grossmann:

- Auslösende Faktoren identifizieren und beseitigen: Dehydratation, Elektrolytverschiebung, metabolische Störung, zerebrovaskuläres Ereignis, SHT, endokrine Störung, Infekt

- Pausieren von ZNS aktiven Substanzen (wie z. B. tri- und tetrazyklische Antidepressiva)

- Auslöser kann jede Anti-ParkinsonTherapie sein, (Dopaminagonisten haben ein höheres Potenzial als L-Dopa)

- Absetzen der adjuvanten ParkinsonTherapie in dieser Reihenfolge: Anticholinergika - Selegilin - Amantadin - Dopaminagonisten und Entacapon

- ev. Reduktion der L-Dopa-Dosis

- Clozapin soll/Quetiapin kann genutzt werden

- Einstellung auf Cholinesterasehemmer, z. B. Rivastigmin

\section{Korrespondenzadresse

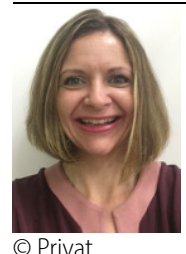 \\ Dr. A. Unger \\ Universitätsklinik für Psychi- atrie und Psychotherapie, Klinische Abteilung für Sozi- alpsychiatrie, Medizinische Universität Wien Währinger Gürtel 18-20, 1090 Wien, Österreich annemarie.unger@ meduniwien.ac.at}

Acknowledgements. Open access funding provided by Medical University of Vienna.

\section{Einhaltung ethischer Richtlinien}

Interessenkonflikt. A. Unger, T. Foki und G. Sachs geben an, dass kein Interessenkonflikt besteht. 
Alle beschriebenen Untersuchungen am Menschen wurden mit Zustimmung der zuständigen EthikKommission, im Einklang mit nationalem Recht sowie gemäß der Deklaration von Helsinki von 1975 (in der aktuellen, überarbeiteten Fassung) durchgeführt. Die Falldarstellung erfolgt vollständig anonymisiert.

Open Access. Dieser Artikel wird unter der Creative Commons Namensnennung 4.0 International Lizenz (http://creativecommons.org/licenses/by/4.0/deed. de) veröffentlicht, welche die Nutzung, Vervielfältigung, Bearbeitung, Verbreitung und Wiedergabe in jeglichem Medium und Format erlaubt, sofern Sie den/die ursprünglichen Autor(en) und die Quelle ordnungsgemäß nennen, einen Link zur Creative Commons Lizenz beifügen und angeben, ob Änderungen vorgenommen wurden.

\section{Literatur}

1. Weintraub D, Chiang C, Kim HM, Wilkinson J, Marras C, Stanislawski B, Mamikonyan E, Kales HC (2016) Association of Antipsychotic use with mortality risk in patients with parkinson disease. JAMA Neurol 73(5):535-541. https://doi.org/10. 1001/jamaneurol.2016.0031

2. Shotbolt P, Samuel M, David A (2010) Quetiapine in the treatment of psychosis in Parkinson's disease. Ther Adv Neurol Disord 3(6):339-350

3. Wilby KJ, Johnson EG, Johnson HE, Ensom MHH (2017) Evidence-based review of Pharmacotherapy used for parkinson's disease psychosis. Ann Pharmacother 51(8):682-695. https://doi.org/10. 1177/1060028017703992

4. Grossmann J, Pirker W (2004) Klinik und Therapie der Parkinson Psychose. J Neurol Neurochir Psychiatr 5(2):42-48

5. Parkinson Study Group (1999) Low-dose clozapine for the treatment of drug-induced psychosis in Parkinson's disease. N Engl J Med 340:757-763

6. Crispo JA, Willis AW, Thibault DP, Fortin Y, Hays HD, McNair DS, Bjerre LM, Kohen DE, Perez-Lloret S, Mattison DR, Krewski D (2016) Associations between Anticholinergic Burden and Adverse Health Outcomes in Parkinson Disease. PLOS ONE 11(3):e150621. https://doi.org/10.1371/journal. pone. 0150621

7. Ballard $C$, Isaacson $S$, Mills $R$, Williams $H$, Corbett A, Coate B, Pahwa R, Rascol O, Burn DJ (2015) Impact of current Antipsychotic medications on comparative mortality and adverse events in people with parkinson disease psychosis. J Am Med Dir Assoc 16(10):898.e1-898.e7. https://doi. org/10.1016/j.jamda.2015.06.021

8. Pollak P, Tison F, Rascol O (2004) Clozapine in drug induced psychosis in Parkinson's disease: a randomised, placebo controlled study with open follow up. J Neurol Neurosurg Psychiatr 75:689-695

9. Factor SA, Friedman JH, Lannon MC, Oakes D, Bourgeois K, Parkinson Study Group. (2001) Clozapine for the treatment of drug-induced psychosis in Parkinson's disease: results of the 12 week open label extension in the PSYCLOPS trial. Mov Disord 16(1):135-139

10. Ondo WG, Tintner R, Voung KD, Lai $D$, Ringholz G (2005) Double-blind, placebo-controlled, unforced titration parallel trial of quetiapine for dopaminergic-induced hallucinations in Parkinson's disease. Mov Disord 20:958-963

11. Rabey JM, Prokhorov T, Miniovitz A, Dobronevsky $\mathrm{E}$, Klein C (2007) Effect of quetiapine in psychotic Parkinson's disease patients: a double-blind labeled study of 3 months' duration. Mov Disord 22:313-318

12. Shotbolt P, Samuel M, Fox C, David AS (2009) A randomized controlled trial of quetiapine for psychosis in Parkinson's disease. Neuropsychiatr Dis Treat 5:327-332

13. Fernandez HH, Okun MS, Rodriguez RL (2009) Quetiapine improves visual hallucinations in Parkinson disease but not through normalization of sleep architecture: results from a doubleblind clinical-polysomnography. Int J Neurosci 119:2196-2205

14. Morgante L, Epifanio A, Spina E, Zappia M, Di Rosa AE, Marconi R, Basile G, Di Raimondo G, La Spina P, Quattrone A (2004) Quetiapine and clozapine in parkinsonian patients with dopaminergic psychosis. Clin Neuropharmacol 27(4):153-156 (Erratum in: Clin Neuropharmacol. 2004 Sep-Oct;27(5):256)

15. Merims D, Balas M, Peretz C, Shabtai H, Giladi N (2006) Rater-blinded, prospective comparison: quetiapine versus clozapine for Parkinson's disease psychosis. Clin Neuropharmacol 29(6):331-337

16. Breier A, Sutton VK, Feldman PD (2002) Olanzapine in the treatment of dopamimetic-induced psychosis in patients with Parkinson's disease. Biol Psychiatry 52:438-445

17. Ondo WG, Levy JK, Vuong KD, Hunter C, Jankovic $J$ (2002) Olanzapine treatment for dopaminergicinduced hallucinations. Mov Disord 17:1031-1035

18. Nichols MJ, Hartlein JM, Eicken MG, Racette BA, Black KJ (2013) A fixed-dose randomized controlled trial of olanzapine for psychosis in Parkinson disease. F1000Res 2:150

19. Meltzer HY, Mills R, Revell S (2010) Pimavanserin, a serotonin(2A) receptor inverse agonist, for

Hier steht eine Anzeige.

\section{Springer}

the treatment of Parkinson's disease psychosis. Neuropsychopharmacology 35:881-892. https:// doi.org/10.1038/npp.2009.176

20. Cummings J, Isaacson S, Mills R (2014) Pimavanserin for patients with Parkinson's disease psychosis: a randomised, placebo-controlled phase 3 trial. Lancet 383:533-540

21. Olsen RE, Kroken RA, Bjørhovde S, Aanesen K, Jørgensen HA, Løberg EM, Johnsen E (2016) Influence of different second generation antipsychotics on the QTc interval: A pragmatic study. World J Psychiatry 6(4):442-448. https://doi.org/10.5498/ wjp.v6.i4.442

22. Fabbri C, Boriani G, Diemberger I, Filippi MG, Ravegnini G, Hrelia P, Minarini A, Albani D, Forloni G, Angelini S, Serretti A (2016) Electrocardiogram Alterations Associated With Psychotropic Drug Use and CACNA1C Gene Variants in Three Independent Samples. Basic Clin Pharmacol Toxicol. https://doi. org/10.1111/bcpt.12720

23. Kasper S, Sachs G (2016) Schizophrenie Medikamentöse Therapie - Konsensus Statement - state of the art 2016. Neuropsy Clinicum, Sonderausgabe Nov. 2016

24. Buchanan RW, Kreyenbuhl J, Kelly DL, Noel JM, Boggs DL, Fischer BA, Himelhoch S, Fang B, Peterson E, Aquino PR, Keller W (2010) The 2009Schizophrenia PORT Psychopharmacological Treatment Recommendations and Summary Statements. Schizophr Bull 36(1):71-93. https:// doi.org/10.1093/schbul/sbp116

25. Pirker W, Fischer P (2003) Cholinesterase-Hemmer in der Therapie der Parkinson-Demenz und der Demenz mit Lewy-Körperchen. J Neurol Neurochir Psychiatr 4(3):6-10 